\title{
Explanations on Divination Instruments by Zhouyi Unearthed in Khara-Khoto
}

\author{
Wei Wang \\ College of History and Culture of Northwest Normal University, Lanzhou Gansu, 730070, China
}

Key words: Khara-Khoto, Divination by Zhouyi, Divinatory symbol.

\begin{abstract}
This paper aims to research the five manuscripts on divinatory symbol by Zhouyi unearthed in Khara-Khoto by catalog, description and explanation respectively, and compare them with the literatures handed down from ancient times. Two of the pieces were jointed together based on the context, and renamed "Thunder-Wind Heng Symbol”.
\end{abstract}

\section{Introduction}

Divination by Zhouyi has a long history in China, which was derived from the square diagram of the 64 hexagrams in the Book of Changes. This divination method has a far-reaching influence on Chinese society. It is one of the most important divination methods in Chinese dynasties, and thrived in the Song Dynasty. Among the manuscripts of divination by Zhouyi unearthed in Khara-Khoto, five manuscripts were found to bear similar contents, and turned out to be from the same manuscript. I will systematically explain and research the five manuscripts one by one.

\section{I (Gen), Symbol of Mountain}

\section{Catalog}

It is collected by the Institute of Cultural Relics and Archaeology, Inner Mongolia, numbered M1-1293[F61:W2]. It is an 18-line manuscript written on bamboo paper in the Yuan Dynasty, which is a little broken and $27.2 \mathrm{~cm}$ high and $18 \mathrm{~cm}$ wide. The content is about the "五 (Gen), symbol of mountain", and each trigram is marked with "stems and branches", "five elements", "six relations" and "self and others" with the traditional "Jing Fang Najia oracles method"; The rest is about the explanations of the divination results. On the upper left is there "seventeen" written with brush in red ink and “ $\mathbf{\Xi}$ (Gen) symbolizes mountain” in big font in black ink, and on the lower right are "A ( $1{ }^{\text {st }}$ heavenly stem)-c ( $3^{\text {rd }}$ earthly branch)-Wood, C-a-Water, C-k-Soil, C-i-Metal, C-g-Fire, C-e-Soil”. The manuscript is circled by brush in red ink.

\section{Explanation}

The figure seventeen on the upper left written with brush in red ink is circled, and “五 (Gen)” exactly ranks seventeenth in the postnatal Eight Trigrams. It is thus clear that this manuscript was sequenced by the order of the postnatal Eight Trigrams, and each trigram is matched with "stems and branches, five elements and six relations". The stems and branches, five elements and six relations were according to the "Jing Fang Najia oracles method".

It is said that the "Jing Fang Najia" is a divination method created by a famous Yi-ology scholar in the Western Han Dynasty named Jing Fang. This method had been prevailing in the circle of divination, and was recorded in many ancient books on divination. The following is about details of 
this method in combination with the record of Liu Dajun in the Najia Oracles Method. The most distinctive feature of the Najia Oracles Method is combining Yi-ology with temperament, and including stems and branches, and five elements in the trigrams, making it the most popular divination method in later generations. According to this method, the 64 hexagrams are divided into

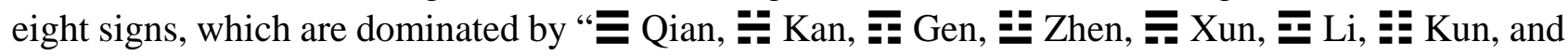
三 Dui” respectively. The Eight Diagrams are called "Pure Eight Diagrams”. Of the eight diagrams, the sign varies from each other, namely mutual transformation between yin and yang, until the upper trigram is changed. Thus, five different signs were produced. The sixth trigram is derived from the fifth changing based on the fourth, which is called "Youhun Trigram". Of the seventh sign, the three trigrams all change, and return to the original position, which is called "Guihun Trigram”. Through changes in this way based on the pure trigram, seven signs were produced, which, plus the pure trigram based on which changes were made, were called the Eight Diagrams. "Najia" means dividing the ten heavenly stems "A, B, C, D, E, F, G, H, I, J" into the 64 hexagrams. The specific practice is “A” for inner three trigrams of $三$ Qian, "I” for outer three trigrams of $三$ Qian; "B” for inner three trigrams of $\mathbf{\Xi}$ Kun, and "J" for outer three trigrams of $\mathbf{\Xi}$ Kun; "C" for both inner and outer three trigrams of $\mathbf{\Xi}$ Gen; “D” for both inner and outer three trigrams of $\mathbf{E}$ Dui; “E” for both inner and outer three trigrams of $\mathbf{- 2}$ Kan; "F” for both inner and outer three trigrams of $\mathbf{\mathbf { Z }} \mathrm{Li}$; “G” for both inner and outer three trigrams of $\mathbf{\Xi}$ Zhen; and " $\mathrm{H}$ ” for both inner and outer three trigrams of $\mathbf{\mathbf { Z }}$ Xun. "A" is the first heavenly stem. Other heavenly stems are included based on "A", so it is called "Najia”.

According to the Najia oracles method, besides the ten heavenly stems, the twelve earthly branches also are included in the Eight Diagrams in such a way: for $\mathbf{E}$ Qian, $\mathbf{\Xi}$ Zhen, $\mathbf{\mathbf { z }}$ Kan, and $\mathbf{\Xi}$ Gen, the branches are arranged clockwise from the first trigram, starting from “a” in 三 Qian and $\mathbf{\Xi}$

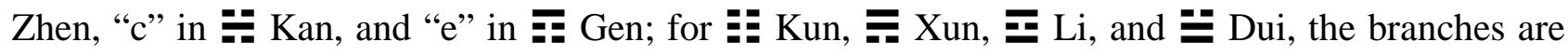

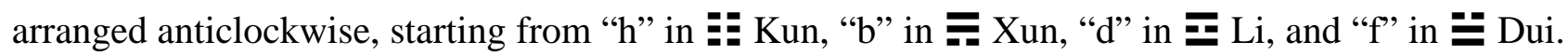
After this, each trigram can be marked with one of the five elements matching therewith.

After the five elements are determined, "six relations" can be determined based on the five elements and the correlations therebetween. If the element in correspondence with the trigram reinforces that with the sign, it relates to parents; if the element in correspondence with the trigram restrains that with the sign, it relates with brothers; that restrains the element in correspondence with the sign relates to official ghost; otherwise relates to offsprings; the one restrained by the element in correspondence with the sign relates to wife and wealth. (The five element arrangement in correspondence with the earthly branches is: a-water, b-soil, c-wood, d-wood, e-wood, f-fire, g-fire, h-soil, i-metal, j-metal, k-soil, l-water. The five elements arrangement in correspondence with the eight signs is: Qian-metal, Zhen-wood, Kan-water, Gen-soil, Kun-soil, Xun-wood, Li-fire and Dui-metal.) The six trigrams of one diagram stand for "self" and "associated" respectively. "Self" refers to the divination seeker, and "associated" refers to others. In each diagram, the positions of "self" and "associated" are different. For the pure sign, the upper trigram refers to "associated", and for other seven signs, the change trigram of the first five signs refers to "associated". The fourth trigram of the "Youhun Trigram" refers to "associated", and the third trigram of the "Guihun Trigram" refers to "associated". After the "associated" is determined, the "self" appears every two trigrams.

Through retrieval and comparison, I haven't found any identical book. Thus, I inferred this manuscript is a transcription of a book having been lost for a long time. However, the content of a poem stated in the end of the manuscript is exactly the same with that stated in the He-Luo Principle-64 Hexagrams Formula. The few different sentences may have been wrongly transcribed. It is thus clear that the manuscript has certain relationship with the He-Luo Principle. The excerpt from the He-Luo Principle is as below:

"Heading northwards and eastwards, every thing goes smoothly.

Things will eventually sort themselves out, and an end means a start." 


\section{Thunder-Heaven, Symbol of Luck}

\section{Catalog}

It is collected by the Institute of Cultural Relics and Archaeology, Inner Mongolia, numbered M1·1294[F62:W23]. It is a 16-line manuscript written on bamboo paper in the Yuan Dynasty, which is a little broken and $27.3 \mathrm{~cm}$ high and $18.2 \mathrm{~cm}$ wide. It is about the explanations of the divination results. On the upper right is there "Thunder-Heaven, Symbol of Luck" in big font in black ink, and "fourteen" written with brush in red ink, and on the lower right are "G-k-soil, G-i-metal, G-g-fire, A-e-soil, A-c-wood, A-a-water”. The manuscript is circled by brush in red ink.

\section{Description}

\section{Explanation}

The figure fourteen on the upper left written with brush in red ink is circled, and "symbol of luck" exactly ranks fourteenth in the postnatal Eight Trigrams. It is thus clear that this manuscript was sequenced by the order of the postnatal Eight Trigrams, and each trigram is matched with "stems and branches, five elements and six relations". The stems and branches, five elements and six relations were according to the "Jing Fang Najia oracles method". The "associated" should be arranged in the fourth and first trigrams, which is identical with the content of the manuscript. Thus, this manuscript is a part of a Jing Fang Najia divination book. The Jing Fang Najia method will be adopted for deduction.

Through retrieval and comparison, I haven't found any identical book. Thus, I inferred this manuscript is a transcription of a book having been lost for a long time. However, the content of two poems stated in the end of the manuscript is not much different from that stated in the He-Luo Principle-64 Hexagrams Formula. The few different sentences may have been wrongly transcribed. It is thus clear that the manuscript has certain relationship with the He-Luo Principle. The excerpt from the He-Luo Principle is as below:

"Being in power in the imperial court and weighing things, things will be made with help.

It is appropriate to make pray, and all excellence grows from here.

Preventive actions should be taken to avoid wealth lost, Weiqing is pessimistic.

Helpful persons finally will be met, and soul communication will be made.

\section{Ben, Symbol of Mountain-Fire}

\section{Catalog}

It is collected by the Institute of Cultural Relics and Archaeology, Inner Mongolia, numbered M1·1295[F61:W3]. It is a 17-line manuscript written on bamboo paper in the Yuan Dynasty, which is a little broken, and $27.7 \mathrm{~cm}$ high and $18.2 \mathrm{~cm}$ wide. It is about the explanations of the divination results. On the upper right is there "Ben, Symbol of Mountain-Fire" in big font in black ink, and on the lower right are "C-c-wood, C-a-water, C-k-soil, F-l-water, F-b-soil, and F-d-wood". The manuscript is circled by brush in red ink.

\section{Description}

Line 1 C-c-wood C-a-water C-k-soil F-l-water F-b-soil F-d-wood

Line 2 Ben, Symbol of Mountain-Fire

Line 3 Get a new lease of life Official Ghost Wife and Wealth Brothers Wife and Wealth Brothers Official Ghost

Line 4 Helpful person Money The skillful

Line 5 Kouyuan Censer Things shine each other

Line 6 Fierce Tiger Rock Manuscript Develop into be skillful 
Line 7 House Ghost Meet some one, and get favor therefrom

Line 8 Symbol Light and heaven should not be neglected

Line 9 After evils are destroyed, luck comes.

Line 10 Walk in dark, and see the dawn finally.

Line 11 Ask for promotion (I) wealth happiness family parents no and body disaster appropriate to pray for gratitude, and get married

Line 12 The dream of making money will be realized, not suitable to give birth, son is weak but will not die, restrains the ancestors

Line 13 Take the opportunity of sacrifice to drive off white tiger

Line 14 A-C-E-G-I Everything is going smoothly.

Line 15 B-D-F-H-J It will rain, and the things you dream of will come true.

Line 16 Salary raise will come, and everything is going smooth.

Line 17 The anxiousness will go away.

\section{Explanation}

Though the manuscript bears no figure, each trigram is matched with "stems and branches, five elements and six relations". The stems and branches, five elements and six relations were according to the "Jing Fang Najia oracles method", but the "associated" is not marked.

It is identical with the content of the manuscript. Thus, this manuscript is a part of a Jing Fang Najia divination book. The Jing Fang Najia method will be adopted for deduction. In the manuscript, detailed explanations of Ben are set forth, relating to promotion, wealth, disease, proceedings, marriage, birth and ancestors, and how to turn ill luck into luck by means of sacrifice, of high practical significance.

Through retrieval and comparison, I haven't found any identical book. Thus, I inferred this manuscript is a transcription of a book having been lost for a long time. However, the content of two poems stated in the end of the manuscript is almost identical with that stated in the He-Luo Principle-64 Hexagrams Formula regardless of the sequence. The few different sentences may have been wrongly transcribed. It is thus clear that the manuscript has certain relationship with the He-Luo Principle. The excerpt from the He-Luo Principle is as below:

"Salary raise will come, and everything is going smooth.

The anxiousness will go away.

Everything is going smoothly.

It will rain, and the things you dream of will come true."

\section{Thunder-Wind Heng Symbol (“Symbol Piece”)}

\section{Catalog}

Two manuscripts earthed from Khara-Khoto are named symbol piece. The first is collected by the Institute of Cultural Relics and Archaeology, Inner Mongolia, numbered M1·1301[F61:W1]. It is an 8-line manuscript written on bamboo paper in the Yuan Dynasty, which is a little broken, and $11.5 \mathrm{~cm}$ high and $6.0 \mathrm{~cm}$ wide. It bears some words like "official ghost, parents, wife and wealth", etc.

\section{Description}

Line $1 \mathrm{H}$-j-metal H-l-water

Line 2 Self Associated

Line 3 Official ghost Parents Wife and wealth

Line 4 Earthly laws

Line 5 Virtuous man has everything go smoothly.

Line 6 Appropriate to stay with the one you meet.

Line 7 No thing is changed. 
Line 8 Lucky

\section{Catalog}

The second is collected by the Institute of Cultural Relics and Archaeology, Inner Mongolia, numbered M1·1302[F62:W22]. It is a 9-line manuscript written on bamboo paper in the Yuan Dynasty, which is a little broken and $6.5 \mathrm{~cm}$ high and $13.4 \mathrm{~cm}$ wide. It bears some words like "getting favor from others", "soar in west wind" etc.

\section{Description}

Line Weidong

Line 2 Clean

Line 3 House

Line 4 Prevent lying-in woman from

Line 5 getting ill.

Line 6 Getting favor from others.

Line 7 It is dangerous and difficult to

Line 8 Phoenix leading young birds.

Line 9 Soar in west wind.

\section{Explanation}

The two manuscripts are similar to the foregoing three manuscripts from the aspects of paper, handwriting, format, etc. Thus, they can be identified to be the same kind of manuscript. But the two manuscripts are heavily broken, and the contents also are not complete. As stated in the first manuscript

"H-j-metal H-l-water

Self Associated

Official ghost Parents”, we can know that official ghost corresponds to j-metal, and parents to l-water, which conforms to the Thunder-Wind Heng Symbol as recorded in the "64 Hexagrams Quick-look Record". Thus, we can infer the two manuscripts relate to the Thunder-Wind Heng Symbol.

The "Phoenix leading young birds", "Soar in west wind" in the second manuscript is similar to the first and the third lines of the He-Luo Principle-64 Hexagrams Formula, "Phoenix leads young birds to fly in the sky, regardless of the long way and twists, soar in west wind, and ascend smoothly". This it can be inferred that the two manuscripts can be joined together, and named Thunder-Wind Heng Symbol according to the principle of naming of the foregoing three manuscripts. (The two manuscripts will be collectively referred to as the Thunder-Wind Heng Symbol below.)

\section{Conclusion}

The five manuscripts were written on bamboo paper in similar handwriting and format, and are well preserved. Hence, they are thought to be from a same category of literature or different pages of the same book. As set forth above, these manuscripts were transcribed from different divination books. The divination method referred to therein should be combinations of different divination methods. I think two reasons causing this phenomenon. Firstly, the divination by Zhouyi may have not been completely established at that time. Secondly, some books relating to divination by Zhouyi including the He-Luo Principle were spread in the Yuan Dynasty, or fortune-tellers transcribed the contents of multiple books to make divination more mysterious and facilitate divination activities.

\section{Acknowledgments}

Title: Research on Effect of Shaman Ceremony in Imperial Court on National Fusion and Cultural Identity in the Early Qing Dynasty, No.:15XJC770002 


\section{References}

[1] CONANT.COM.CN, M1·1293[F61:W2], see Volume VIII of the Literatures of Chinese Collections - Khara-Khoto for the plate, p1593.

[2] Liu Dajun: Najia Oracles Method, QiLu Press, 1995.

[3] Chen Tuan (Writer), Shao Yong (Narrator): He-Luo Principle, QiLu Press, 1995, p737.

[4] CONANT.COM.CN, M1·1294[F62:W23], see Volume VIII of the Literatures of Chinese Collections-Khara-Khoto for the plate, p1614.

[5] Chen Tuan (Writer), Shao Yong (Narrator): He-Luo Principle, QiLu Press, 1995, p739.

[6] CONANT.COM.CN, M1·1301[F61:W1], see Volume VIII of the Literatures of Chinese Collections-Khara-Khoto for the plate, p1620.

[7] Xu: Divination Orthodox, Qinghai People’s Publishing House, 1991, p14.

[8] Chen Tuan (Writer), Shao Yong (Narrator): He-Luo Principle, QiLu Press, 1995, p737. 